\title{
Epidemiology of Streptococcus pneumoniae and Staphylococcus aureus colonization in healthy Venezuelan children
}

\author{
B. Quintero - M. Araque - C. van der Gaast-de Jongh • \\ F. Escalona - M. Correa - S. Morillo-Puente • \\ S. Vielma • P. W. M. Hermans
}

Received: 1 April 2010 /Accepted: 10 August 2010/Published online: 30 August 2010

(C) The Author(s) 2010. This article is published with open access at Springerlink.com

\begin{abstract}
Streptococcus pneumoniae and Staphylococcus aureus cause significant morbidity and mortality worldwide. We investigated both the colonization and co-colonization characteristics for these pathogens among 250 healthy children from 2 to 5 years of age in Merida, Venezuela, in 2007. The prevalence of $S$. pneumoniae colonization, $S$. aureus colonization, and $S$. pneumoniae-S. aureus cocolonization was $28 \%, 56 \%$, and $16 \%$, respectively. Pneumococcal serotypes $6 \mathrm{~B}(14 \%), 19 \mathrm{~F}(12 \%), 23 \mathrm{~F}(12 \%), 15(9 \%)$, $6 \mathrm{~A}(8 \%), 11(8 \%), 23 \mathrm{~A}(6 \%)$, and $34(6 \%)$ were the most prevalent. Non-respiratory atopy was a risk factor for $S$. aureus colonization $(p=0.017)$. Vaccine serotypes were negatively
\end{abstract}

B. Quintero $\cdot$ S. Vielma

Department of Microbiology and Parasitology,

Faculty of Medicine, Los Andes University,

Mérida, Venezuela

B. Quintero • C. van der Gaast-de Jongh • P. W. M. Hermans ( $\triangle)$

Laboratory of Pediatric Infectious Diseases,

Radboud University Nijmegen Medical Centre,

P.O. Box 9101, 6500 HB Nijmegen, The Netherlands

e-mail: P.Hermans@cukz.umcn.nl

M. Araque

Department of Microbiology and Parasitology,

Faculty of Pharmacy and Bioanalysis,

Laboratory of Molecular Microbiology, Los Andes University,

Mérida, Venezuela

F. Escalona $\cdot$ M. Correa

Los Andes University Hospital,

Mérida, Venezuela

S. Morillo-Puente

Department of Methodology,

Faculty of Legal and Political Sciences, Los Andes University,

Mérida, Venezuela associated with preceding respiratory infection $(p=0.02)$ and with $S$. aureus colonization $(p=0.03)$. We observed a high prevalence of pneumococcal resistance against trimethoprimsulfamethoxazole $(40 \%)$, erythromycin $(38 \%)$, and penicillin (14\%). Semi-quantitative measurement of pneumococcal colonization density showed that children with young siblings and low socioeconomic status were more densely colonized ( $p=0.02$ and $p=0.02$, respectively). In contrast, trimethoprimsulfamethoxazole- and multidrug-resistant-pneumococci colonized children sparsely ( $p=0.03$ and $p=0.01$, respectively). Our data form an important basis to monitor the future impact of pneumococcal vaccination on bacterial colonization, as well as to recommend a rationalized and restrictive antimicrobial use in our community.

\section{Introduction}

Streptococcus pneumoniae is one of the major pathogens infecting humans worldwide. It is the most common cause of community-acquired bacterial pneumonia and otitis media, but can also give rise to severe cases of meningitis and sepsis. It is estimated that 1.6 million people die from pneumococcal diseases every year [1]. Despite causing severe diseases, $S$. pneumoniae is also asymptomatically carried in the nose, nasopharynx, and throat. In children, the isolation rates of $S$. pneumoniae obtained by nasal and nasopharyngeal sampling are similar, but higher than by oropharyngeal sampling [2,3]. The prevalence of $S$. pneumoniae nasopharyngeal colonization varies from 7 to $99 \%$, depending on the age, health, and socioeconomic status of the study population [4].

Although there are currently over 90 distinct serotypes, certain serotypes commonly account for the majority of 
S. pneumoniae nasopharyngeal isolates. However, the distribution of serotypes is temporal and varies according to geographic location. The serotype distribution among carriage isolates is often used as an indicator for theoretical vaccine coverage [4]. Several clinical and demographic characteristics have been positively associated with an increase in $S$. pneumoniae colonization, such as young age, crowding, day care attendance, family size, number of siblings, poverty, smoking, and recent antibiotic use [4, 5]. In particular, the worldwide rise of pneumococcal antibiotic resistance is alarming and represents a threat for the successful treatment of infections caused by this pathogen $[6,7]$.

Staphylococcus aureus is a frequent cause of clinically relevant diseases, ranging from relatively mild infections such as skin infections and otitis media, to life-threatening invasive infections such as pneumonia, bacteremia, and endocarditis. S. aureus is also asymptomatically carried on the skin, in the perineum, nose, and nasopharynx. Although multiple body sites can be colonized, $S$. aureus is most frequently carried in the anterior nares of the nose. S. aureus is carried by approximately $30 \%$ of healthy adults and $10 \%$ of children $[8,9]$. Older siblings, family size, breast-feeding, and passive smoking have been suggested to influence the $S$. aureus carriage rate in healthy children $[9,10]$. Moreover, several studies have shown increased $S$. aureus nasal colonization among patients with respiratory and nonrespiratory allergies compared to the general population $[11,12]$. In addition, symptomatic allergic rhinitis leads to an increase in the airborne dispersal of $S$. aureus $[13,14]$.

Epidemiological studies monitoring the carriage of $S$. pneumoniae and $S$. aureus are important for several reasons. First, colonization in healthy individuals is a prerequisite for developing invasive and non-invasive diseases, and reduced colonization has been correlated with decreased pneumococcal and staphylococcal infection rates $[4,15]$. Second, healthy carriers serve as reservoirs for $S$. aureus and $S$. pneumoniae transmission to others in the community and in the hospital [8-10]. And third, carriage strains have been used as indicators for drug resistance, and for pneumococcal serotype distribution and vaccine coverage prediction [6].

Bacterial colonization is also the result of interspecies competition, and some bacterial species are either positively or negatively associated during co-colonization. For instance, several epidemiologic studies have reported a negative association between $S$. pneumoniae nasopharyngeal colonization and $S$. aureus nasal colonization, which was most significant for S. pneumoniae serotypes included in the 7valent pneumococcal conjugate vaccine $[10,16,17]$. However, the epidemiology of co-colonization for these pathogens in the same ecological niche, i.e., the nose of healthy children, has not been investigated in detail.
The importance of $S$. pneumoniae carriage surveillance in Latin American countries is underlined by the significant contribution of this pathogen to pediatric morbidity and mortality. The population of Latin America in 2007 was 565 million [18], and the estimated annual burden of pneumonia, meningitis, and otitis media caused by $S$. pneumoniae in children under 5 years of age are in the ranges 980,000 $1,500,000,2,600-6,800$, and $980,000-1,500,000$, respectively [1]. Even though an estimated 12,000 to 28,000 deaths due to pneumococcal disease occur in the region annually, infant pneumococcal vaccination is not a routine part of the current immunization programs in any of the countries in Latin America [1].

Information regarding the distribution of pneumococcal serotypes, as well as clinical and demographic characteristics associated with $S$. aureus and $S$. pneumoniae colonization, are important for the design and monitoring of strategies to prevent and control these infections $[4,15]$. However, in Venezuela, data regarding the serotype distribution and prevalence of $S$. pneumoniae colonization and epidemiological records of nasal colonization of $S$. aureus in healthy children is scarce [19].

The objective of this study was to investigate in detail the microbiological, clinical, and demographic features associated with $S$. pneumoniae and $S$. aureus nasal colonization and co-colonization among healthy Venezuelan children.

\section{Materials and methods}

\section{Study population}

All children enrolled in this study were sampled in January and February 2007 in five schools in Merida, Venezuela. Criteria for inclusion were age under 5 years, absence of pneumococcal vaccination, and no current respiratory infection (RI). To avoid the inclusion of children with possible current RI, we excluded children showing symptoms or signs of RI, as well as those who received at least one dose of any antibiotic treatment during the previous 15 days. One of the children's parents signed informed consent and provided clinical and demographic information, such as age, sex, crowding (i.e., two or more people sleeping in the same room), siblings, smokers at home, and socioeconomic status. In addition, we asked whether the children also attended a day care center when they were not at school. Detailed medical information was recorded, such as previous diagnosis of atopies, asthma, allergic rhinitis and non-respiratory atopies, history of hospitalization in the previous 6 months, and antibiotic treatment in the previous 3 months. Incidence and history of previous RIs were also recorded in detail. The study was reviewed and approved 
by the ethical committees of the Council for Scientific, Humanistic and Technological Development (CDCHT) of the Los Andes University (Mérida, Venezuela).

Sample collection

Mucus specimens from both nares of the child were obtained using a sterile cotton-tipped swab. Swabs were plated immediately onto brain heart infusion agar plates with $5 \%$ blood and $4 \mu \mathrm{g} / \mathrm{ml}$ of gentamycin, and onto mannitol salt agar plates (Oxoid ${ }^{\circledR}$, Badhoevedorp, The Netherlands) by rolling the swab over one-quarter of the plate and streaking the sample onto four quadrants using a sterile loop as previously described [20]. Blood agar plates and mannitol agar plates were then incubated at $35^{\circ} \mathrm{C}$ under microaerophilic and aerobic conditions, respectively.

Bacterial isolation, identification, and serotyping

S. pneumoniae was identified by Gram stain, colony characteristics, susceptibility to optochin, and bile solubility. Pneumococcal serotypes were determined by the capsular reaction test (Quellung reaction) using specific antisera (Statens Serum Institut, Copenhagen, Denmark). Six strains were not available for serotyping. For the serogroups 10, 11, and 15, antiserum for subtyping was not available. All pneumococcal Quelling-non-typeable strains were reinvestigated for the presence of the most common pneumococcal serotypes using a multiplex polymerase chain reaction (PCR) assay as described previously [21]. Additionally, for all 6A serotypes and non-typeable strains, we determined by PCR the presence of wciN6C of serotype $6 \mathrm{C}$ as described previously [22]. The 6C capsular type was confirmed by Dr. Moon Nahm (University of Alabama, Birmingham, AL, USA) using 6C-specific monoclonal anticapsule antibodies (Hyp6AM3) as previously described [23]. Those serotypes included in the heptavalent pneumococcal polysaccharide conjugate vaccine (Prevenar ${ }^{\circledR}$, Wyeth Pharmaceuticals, Philadelphia, PA, USA) were considered as vaccine serotypes. $S$. aureus was identified by Gram stain, colony characteristics, and biochemical conventional tests.

\section{Susceptibility testing}

S. pneumoniae susceptibility and minimal inhibitory concentrations (MICs) for 19 antimicrobial drugs were determined following the recommendations of the Clinical and Laboratory Standards Institute (CLSI) guidelines [24]. Six $S$. pneumoniae strains were not available for susceptibility testing. For all MIC testing, S. pneumoniae ATCC 49619 was included as a quality control strain. The agar dilution method was performed to determine penicillin susceptibility using Penicillin G $\left(\right.$ Calbiochem ${ }^{\circledR}$, Merck, Darmstadt,
Germany) and Mueller-Hinton II agar (BBL Microbiology Systems, Cockeysville, MD, USA) supplemented with $5 \%$ sheep blood. For the other 18 antimicrobials, we used a commercial microdilution panel (Sensititre ${ }^{\circledR}$ STP5FStreptococcus pneumoniae MIC plate, TREK Diagnostic Systems, East Grinstead, UK) containing cefepime, cefotaxime, ceftriaxone, cefuroxime, clindamycin, daptomycin, erythromycin, ertapenem, levofloxacin, linezolid, meropenem, moxifloxacin, chloramphenicol, tigecycline, tetracycline, telithromycin, trimethoprim-sulfamethoxazole, and vancomycin. We used Mueller-Hinton agar supplemented with lysed horseblood broth (CP112-10, TREK Diagnostic Systems, East Grinstead, UK) as the test medium. The MIC breakpoints suggested by the current CLSI 2009 document were used for all antimicrobials tested. In the case of penicillin and cefuroxime, we used the breakpoints suggested for oral treatment (penicillin: S: susceptible, $\leq 0.06 \mu \mathrm{g} / \mathrm{ml}$; I: intermediate, $0.12-1 \mu \mathrm{g} / \mathrm{ml}$; R: resistant, $\geq 2 \mu \mathrm{g} / \mathrm{ml}$; and cefuroxime: $\mathrm{S}, \leq 1 \mu \mathrm{g} / \mathrm{ml} ; \mathrm{I}, 2 \mu \mathrm{g} / \mathrm{ml} ; \mathrm{R}, \geq 4 \mu \mathrm{g} / \mathrm{ml})$. For the other cephalosporins, i.e., cefotaxime, ceftriaxone and cefepime, the breakpoints suggested for non-meningitis were used (S: $\leq 1 \mu \mathrm{g} / \mathrm{ml} ;$ I: $2 \mu \mathrm{g} / \mathrm{ml} ; \mathrm{R}: \geq 4 \mu \mathrm{g} / \mathrm{ml}$ ). We considered strains being non-susceptible to three or more different classes of antimicrobials as multidrug-resistant (MDR).

\section{Colonization density of $S$. pneumoniae}

We used the number of colony forming units (CFUs) in $S$. pneumoniae-positive cultures as an indicator for colonization density. All S. pneumoniae-positive cultures were checked after 20-24 h of incubation, and the CFUs were counted by the same operator. CFUs were scored using a semi-quantitative scale by O'Brien et al. [20] as follows: scant growth: $<25$ CFUs in quadrant $1 ; 1+$ growth: $\geq 25$ CFUs in quadrant 1 and $<25$ in quadrant $2 ; 2+$ growth: $\geq 25$ CFUs in quadrant 2 and $<25$ in quadrant $3 ; 3+$ growth: $\geq 25$ CFUs in quadrant 3 and $<25$ in quadrant 4 ; and 4+ growth: $\geq 25$ CFUs in quadrant 4 . For statistical analyses, we classified children into two groups: sparsely colonized (if bacterial growth was scant, $1+$, or $2+$ ) or densely colonized (if bacterial growth was $3+$ or $4+$ ) with S. pneumoniae.

Statistical analysis

All statistical analyses were performed using Windows Statistical Package for the Social Sciences, v.17.0. The association between $S$. pneumoniae colonization, S. aureus colonization, and all other variables, i.e., clinical and epidemiological parameters of children and microbiological characteristics of $S$. pneumoniae, was determined using univariate and multivariate statistical analyses. Fisher's exact test was used for all univariate analyses, except when 
the variables had more than two categories. For the latter, we applied the Pearson's Chi-square statistical analysis. All statistical tests were two-tailed and $p$-values $<0.05$ were considered to be significant. Those variables with $p$-values of 0.15 or less in univariate analyses were then included in a multivariate analysis, i.e., multiple logistic binary regression analysis.

\section{Results}

The study population included 250 healthy children aged 2 to 5 years (average 4.43 years; median 5 years), of which $56 \%$ were female. The distribution of age was as follows: 2 years: 1 child; 3 years: 19 children; 4 years: 101 children; and 5 years: 129 children. Of the children's families, $6 \%$, $84 \%$, and $11 \%$ belonged to high, medium, or low socioeconomic status, respectively. The clinical and demographic characteristics of the children enrolled in this study are listed in Tables 1 and 2.

$S$. pneumoniae and S. aureus were isolated from 71 (28\%) and 141 children (56\%), respectively. Co-colonization of $S$. pneumoniae and $S$. aureus was observed in 39 children (16\%) (Table 1). The proportion of $S$. pneumoniae colonization showed a peak at the age of 3 years $(42 \%$; $n=$ $8 / 19)$, whereas a decrease of the prevalence among older children was observed (4 years: $29 \%, n=29 / 101$, and 5 years: $26 \%, n=34 / 129$ ). Instead, the frequency of $S$. aureus colonization scarcely varied between different age groups: $53 \%(10 / 19)$ among children 3 years of age, 55\% (56/101) among children 4 years of age, and 58\% (75/129) among children 5 years of age. No colonization by $S$. pneumoniae nor by $S$. aureus was detected in the children who were 2 years of age. The univariate analysis showed that the occurrence of colonization with $S$. pneumoniae or $S$. aureus was not statistically different between children aged 4 years or less versus children of age 5 years $(p=0.48$ and $p=0.61$, respectively; Table 1). The highest rates of $S$. pneumoniae colonization were observed among children with siblings $(32 \%)$, with siblings $\leq 5$ years old $(35 \%)$, and with frequent RI $(46 \%)$. In contrast, the lowest prevalence rates were observed among asthmatic children (21\%) and among children attending a day care center (21\%) (Tables 1 and 2). However, none of these factors were positively or negatively associated with $S$. pneumoniae colonization. The highest prevalence rates of $S$. aureus colonization were observed among children living in crowding (73\%) and having non-respiratory atopies (75\%), whereas the lowest prevalence rates were observed among asthmatic children (44\%) (Tables 1 and 2). However, the occurrence of non-respiratory atopies was the only characteristic positively associated with $S$. aureus colonization ( $p=0.018$ [univariate analysis] and $p=0.017$ [multivariate analysis], respectively; Table 1).
S. pneumoniae-S. aureus co-colonization according to the distribution of age was as follows: 3 years $26 \%(5 / 19$ children), 4 years $17 \%$ (17/101 children), and 5 years $13 \%$ $(17 / 129)$. According to the univariate analysis, the occurrence of $S$. pneumoniae-S. aureus co-colonization was not statistically different between children aged 4 years or less versus children 5 years of age $(p=0.29$; Table 1$)$. We observed that the proportion of $S$. pneumoniae-S. aureus co-colonization was higher among children with siblings compared to those without siblings $(19 \%$ vs. $8 \%$, respectively), but lower among asthmatic children compared to non-asthmatic children ( $5 \%$ vs. $18 \%$, respectively; Tables 1 and 2). However, none of these factors were statistically associated with the $S$. pneumoniae-S. aureus co-colonization using univariate or multivariate analyses.

The $S$. pneumoniae strains isolated in this study displayed 16 different pneumococcal serotypes, of which 6B (14\%), 19F (12\%), 23F (12\%), 15 (9\%) 6A (8\%), 11 $(8 \%), 23 \mathrm{~A}(6 \%)$, and $34(6 \%)$ were the most common. Serotypes 14 and 38 were found in three strains (5\%) and serotypes $6 \mathrm{C}, 9 \mathrm{~N}, 10,18 \mathrm{~A}, 20$, and $23 \mathrm{~B}$ were found in only one strain $(1 \%)$. Six percent of the strains $(n=4)$ were non-typeable. The serotype $6 \mathrm{C}$ strain did not react with any pneumococcal antiserum during the Quellung test and it was initially classified as non-typeable. However, we did detect the wciN6C gene by PCR in this strain, and the $6 \mathrm{C}$ capsular type was confirmed using 6C-specific monoclonal anticapsule antibodies.

In total, 28 out of the $65 S$. pneumoniae strains (43\%) displayed vaccine serotypes. The prevalence of colonization of $S$. pneumoniae vaccine serotypes and the clinical demographic characteristics are given in the Table 3 . We observed that children with a previous RI were less often colonized by vaccine serotypes than children without previous RI (18\% vs. $52 \%$, respectively). According to the univariate, but not to the multivariate, analysis, having a previous RI was a factor inversely associated with vaccine serotype colonization ( $p=0.02$ and $p=0.057$, respectively).

We observed no differences in the proportion of $S$. pneumoniae isolated from children colonized or not colonized with $S$. aureus ( $28 \%$ vs. $29 \%$, respectively, $p=$ 0.77; Table 1). Likewise, we observed no differences in the proportion of $S$. aureus isolated from children colonized or not colonized with $S$. pneumoniae (55\% vs. $57 \%$, respectively, $p=0.77$; Table 1). However, the proportion of $S$. aureus isolated among children colonized with vaccine serotypes was nearly two-fold lower than among children colonized with non-vaccine serotypes (36\% vs. $68 \%$, respectively). Likewise, we observed that the proportion of vaccine serotypes isolated among children colonized with $S$. aureus was two-fold lower than among children non-colonized with $S$. aureus (29\% vs.60\%, respectively). The univariate analysis indicated that the colonization with 


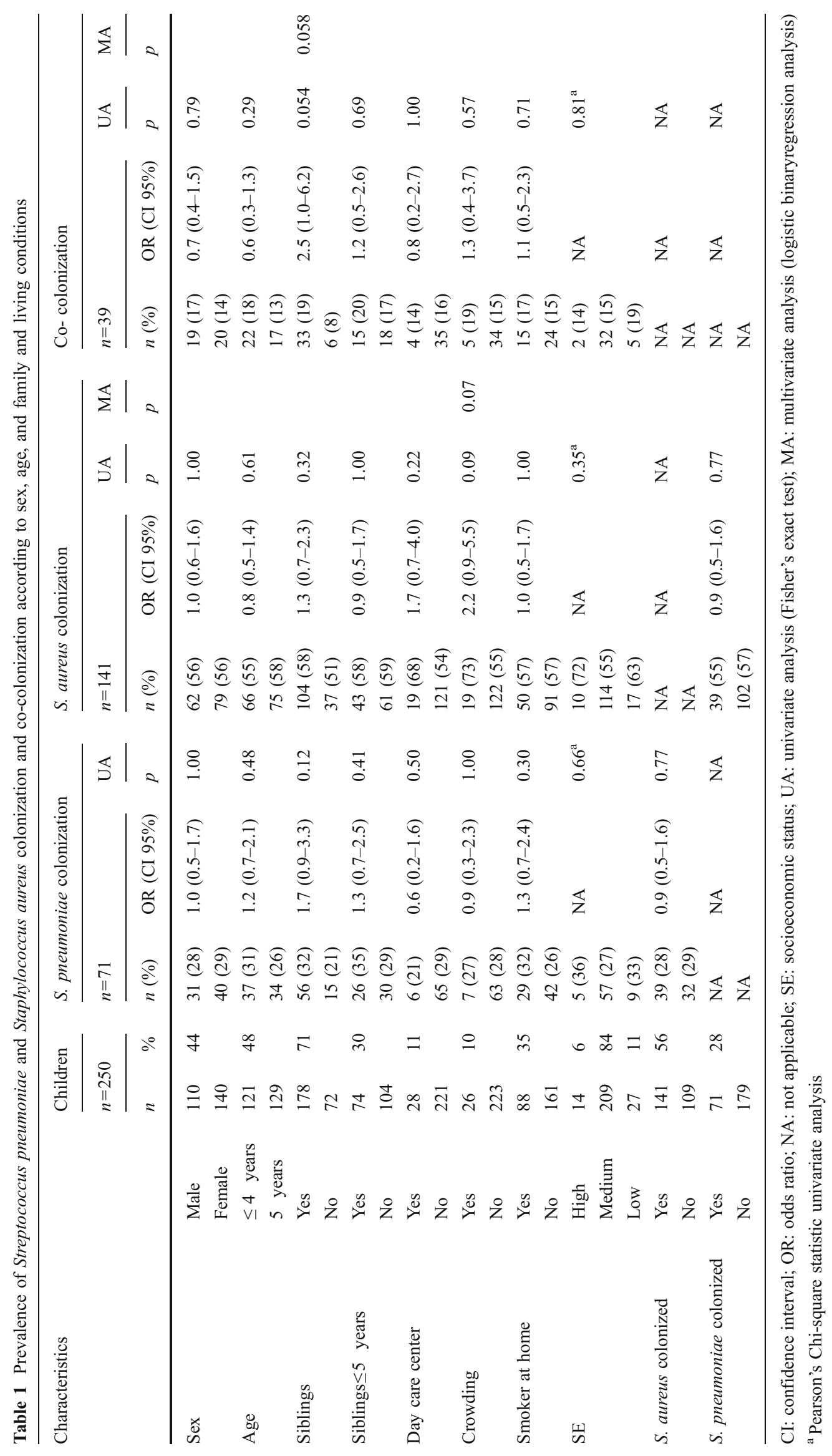




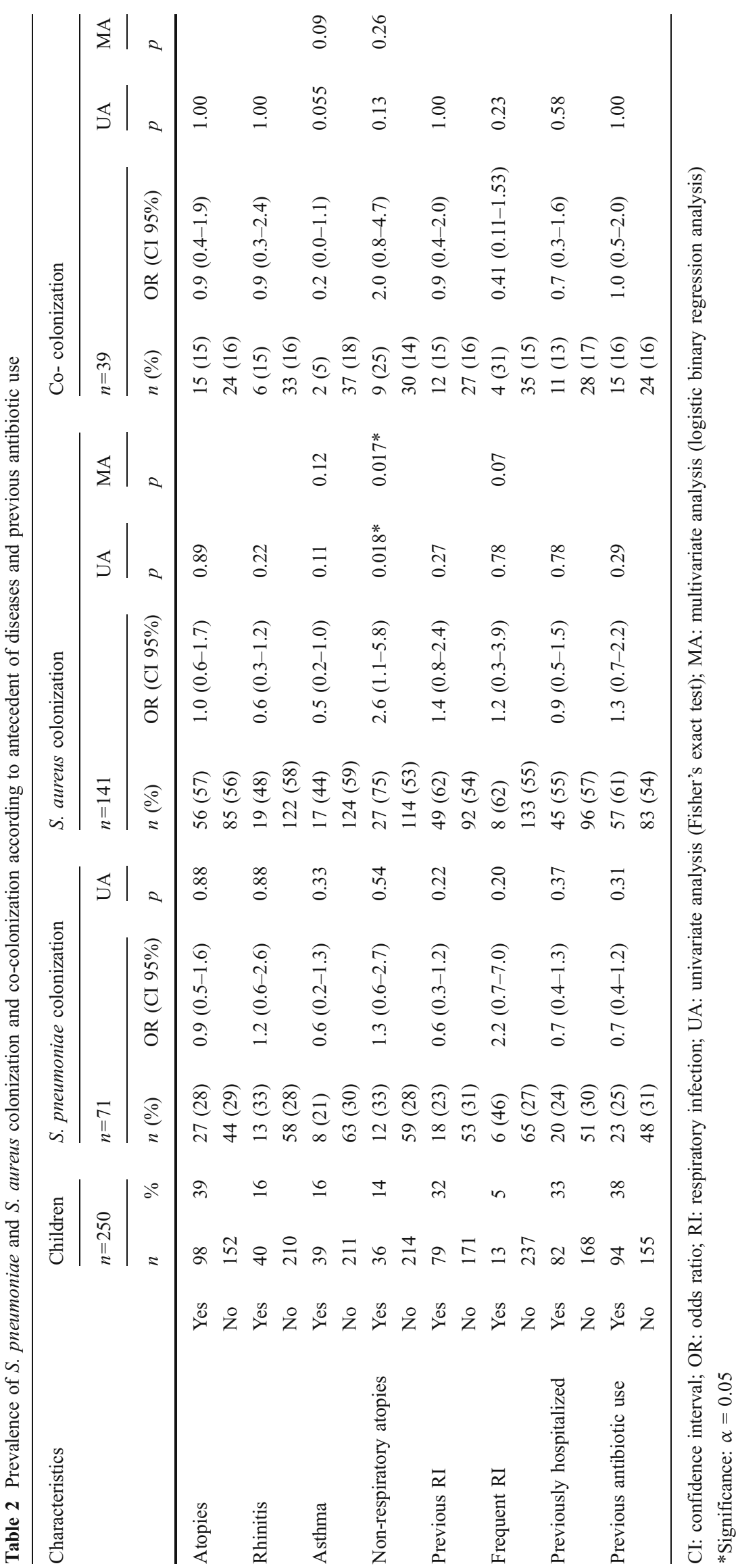


Table 3 Prevalence of S. pneumoniae vaccine serotypes colonization according to the clinical demographic characteristics

\begin{tabular}{|c|c|c|c|c|c|c|c|}
\hline \multirow[t]{2}{*}{ Characteristics } & & \multirow{3}{*}{$\begin{array}{l}\text { S. pneumoniae } \\
\text { isolated strains } \\
n \\
71\end{array}$} & \multirow{3}{*}{$\begin{array}{l}\text { S. pneumoniae } \\
\text { serotyped strains } \\
n \\
65\end{array}$} & \multicolumn{4}{|c|}{ Vaccine serotypes } \\
\hline & & & & \multirow{2}{*}{$\frac{n(\%)^{\mathrm{a}}}{28(43)}$} & \multirow[b]{2}{*}{ OR (CI 95\%) } & \multirow{2}{*}{$\frac{\mathrm{UA}}{p}$} & \multirow{2}{*}{$\frac{\mathrm{MA}}{p}$} \\
\hline Total & & & & & & & \\
\hline \multirow[t]{2}{*}{ Sex } & Male & 31 & 29 & $16(55)$ & $0.4(0.1-1.1)$ & 0.08 & 0.07 \\
\hline & Female & 40 & 36 & $12(33)$ & & & \\
\hline \multirow[t]{2}{*}{ Siblings } & Yes & 56 & 52 & $21(40)$ & $0.5(0.1-1.9)$ & 0.53 & \\
\hline & No & 15 & 13 & $7(54)$ & & & \\
\hline \multirow[t]{2}{*}{ Siblings $\leq 5$ years } & Yes & 26 & 24 & $10(42)$ & $1.1(0.3-3.3)$ & 1.00 & \\
\hline & No & 30 & 28 & $11(39)$ & & & \\
\hline \multirow[t]{2}{*}{ Day care center } & Yes & 6 & 6 & $2(33)$ & $0.6(0.1-3.7)$ & 0.69 & \\
\hline & No & 65 & 59 & $26(44)$ & & & \\
\hline \multirow[t]{2}{*}{ Crowding } & Yes & 7 & 6 & $4(67)$ & $2.8(0.4-16.7)$ & 0.39 & \\
\hline & No & 63 & 58 & $24(41)$ & & & \\
\hline \multirow[t]{2}{*}{ Smoker at home } & Yes & 29 & 27 & $13(48)$ & $1.4(0.5-3.8)$ & 0.61 & \\
\hline & No & 42 & 38 & $15(39)$ & & & \\
\hline \multirow[t]{2}{*}{ Atopies } & Yes & 27 & 24 & $11(46)$ & $1.1(0.4-3.2)$ & 0.79 & \\
\hline & No & 44 & 41 & $17(41)$ & & & \\
\hline \multirow[t]{2}{*}{ Rhinitis } & Yes & 13 & 12 & $5(42)$ & $0.9(0.2-3.3)$ & 1.00 & \\
\hline & No & 58 & 53 & $23(43)$ & & & \\
\hline \multirow[t]{2}{*}{ Asthma } & Yes & 8 & 8 & $5(63)$ & $2.4(0.5-11.3)$ & 0.27 & \\
\hline & No & 63 & 57 & $23(40)$ & & & \\
\hline \multirow[t]{2}{*}{ Non-respiratory atopies } & Yes & 12 & 10 & $5(50)$ & $1.3(0.3-5.3)$ & 0.73 & \\
\hline & No & 59 & 55 & $23(42)$ & & & \\
\hline \multirow[t]{2}{*}{ Previous RI } & Yes & 18 & 17 & $3(18)$ & $0.1(0.0-0.7)$ & $0.02 *$ & 0.057 \\
\hline & No & 53 & 48 & $25(52)$ & & & \\
\hline \multirow[t]{2}{*}{ Frequent RI } & Yes & 6 & 5 & $3(60)$ & $2.1(0.3-13.5)$ & 0.64 & \\
\hline & No & 65 & 60 & $25(42)$ & & & \\
\hline \multirow[t]{2}{*}{ Previously hospitalized } & Yes & 20 & 18 & $11(61)$ & $2.7(0.9-8.4)$ & 0.09 & 0.11 \\
\hline & No & 51 & 47 & $17(36)$ & & & \\
\hline \multirow[t]{2}{*}{ Previous antibiotic use } & Yes & 23 & 21 & $11(52)$ & $1.7(0.6-4.9)$ & 0.42 & \\
\hline & No & 48 & 44 & $17(39)$ & & & \\
\hline \multirow[t]{2}{*}{ S. aureus colonization } & Yes & 39 & 35 & $10(29)$ & $0.2(0.0-0.7)$ & $0.013^{*}$ & $0.03 *$ \\
\hline & No & 32 & 30 & $18(60)$ & & & \\
\hline \multirow[t]{3}{*}{ SE } & High & 5 & 4 & $4(100)$ & NA & $0.048^{\mathrm{b}}$ & 0.61 \\
\hline & Medium & 57 & 53 & $20(38)$ & & & \\
\hline & Low & 9 & 8 & $4(50)$ & & & \\
\hline
\end{tabular}

CI: confidence interval; OR: odds ratio; RI: respiratory infection; SE: socioeconomic status; NA: not applicable; UA: univariate analysis (Fisher's exact test); MA:multivariate analysis (logistic binary regression analysis)

${ }^{\text {a }}$ Percentages were calculated on the basis of the $65 \mathrm{~S}$. pneumoniae strains available for serotyping

${ }^{\mathrm{b}}$ Pearson's Chi-square statistic univariate analysis

*Significance: $\alpha=0.05$

S. pneumoniae vaccine serotypes was negatively associated with $S$. aureus colonization $(p=0.013$; Table 3$)$. This negative relationship remained significant after the multivariate analysis $(p=0.03$; Table 3$)$. In other words, in particular, the vaccine-type pneumococci were a risk factor for $S$. aureus colonization and vice versa.
Colonization with $S$. pneumoniae was dense in $23 \%(n=$ 16) and sparse in $77 \%(n=55)$ of the 71 children with positive cultures (Table 4). A dense colonization by S. pneumoniae was frequently seen among children with a low socioeconomic status $(56 \%)$, attending day care centers $(50 \%)$, with siblings $\leq 5$ years old (35\%), and with a previous RI (39\%) 
Table 4 Colonization density of $S$. pneumoniae and clinical and demographic characteristics of the 71 colonized children

\begin{tabular}{|c|c|c|c|c|c|c|c|}
\hline \multirow[t]{3}{*}{ Characteristics } & & \multirow[t]{3}{*}{$N$} & \multicolumn{2}{|l|}{ Colonization } & \multirow[t]{3}{*}{ OR (CI 95\%) } & \multirow{3}{*}{$\begin{array}{l}\text { UA } \\
p\end{array}$} & \multirow{3}{*}{$\begin{array}{l}\text { MA } \\
p\end{array}$} \\
\hline & & & \multirow{2}{*}{$\begin{array}{l}\text { Sparse bacterial growth } \\
(\text { scant, } 1+, \text { or } 2+) \\
n(\%)\end{array}$} & \multirow{2}{*}{$\begin{array}{l}\text { Dense bacterial growth } \\
(3+\text { or } 4+) \\
n(\%)\end{array}$} & & & \\
\hline & & & & & & & \\
\hline Total & & 71 & $55(77)$ & $16(23)$ & & & \\
\hline \multirow[t]{2}{*}{ Sex } & Male & 31 & $26(84)$ & $5(16)$ & $0.5(0.1-1.6)$ & 0.39 & \\
\hline & Female & 40 & $29(73)$ & $11(28)$ & & & \\
\hline \multirow[t]{2}{*}{ Siblings } & Yes & 56 & $44(79)$ & $12(21)$ & $1.3(0.3-4.9)$ & 0.73 & \\
\hline & No & 15 & $11(73)$ & $4(27)$ & & & \\
\hline \multirow[t]{2}{*}{ Siblings $\leq 5$ years: } & Yes & 26 & $17(65)$ & $9(35)$ & $0.2(0.0-0.8)$ & $0.047^{*}$ & $0.02 *$ \\
\hline & No & 30 & $27(90)$ & $3(10)$ & & & \\
\hline \multirow[t]{2}{*}{ Day care center } & Yes & 6 & $3(50)$ & $3(50)$ & $0.2(0.0-1.3)$ & 0.12 & 0.07 \\
\hline & No & 65 & $52(80)$ & $13(20)$ & & & \\
\hline \multirow[t]{2}{*}{ Crowding } & Yes & 7 & $5(71)$ & $2(29)$ & $0.7(0.1-4.0)$ & 0.65 & \\
\hline & No & 63 & $50(79)$ & $14(22)$ & & & \\
\hline \multirow[t]{2}{*}{ Smoker at home } & Yes & 29 & $22(76)$ & $7(24)$ & $0.8(0.2-2.6)$ & 0.78 & \\
\hline & No & 42 & $33(79)$ & $9(21)$ & & & \\
\hline \multirow[t]{2}{*}{ Atopies } & Yes & 27 & $22(81)$ & $5(19)$ & $1.4(0.4-4.8)$ & 0.57 & \\
\hline & No & 44 & $33(75)$ & $11(25)$ & & & \\
\hline \multirow[t]{2}{*}{ Rhinitis } & Yes & 13 & $10(77)$ & $3(23)$ & $0.9(0.2-4.0)$ & 1.00 & \\
\hline & No & 58 & $45(77)$ & $13(22)$ & & & \\
\hline \multirow[t]{2}{*}{ Asthma } & Yes & 8 & $5(63)$ & $3(38)$ & $0.4(0.0-2.0)$ & 0.36 & \\
\hline & No & 63 & $50(79)$ & $13(21)$ & & & \\
\hline \multirow[t]{2}{*}{ Non-respiratory atopies } & Yes & 12 & $12(100)$ & $0(0)$ & $0.7(0.6-0.8)$ & 0.056 & 0.99 \\
\hline & No & 59 & $43(73)$ & $16(27)$ & & & \\
\hline \multirow[t]{2}{*}{ Previous RI } & Yes & 18 & $11(61)$ & $7(39)$ & $0.3(0.0-1.0)$ & 0.09 & 0.47 \\
\hline & No & 53 & $44(83)$ & $9(17)$ & & & \\
\hline \multirow[t]{2}{*}{ Frequent RI } & Yes & 6 & $6(100)$ & $0(0)$ & $1.3(1.1-1.5)$ & 0.32 & \\
\hline & No & 65 & $49(75)$ & $16(25)$ & & & \\
\hline \multirow[t]{2}{*}{ Previously hospitalized } & Yes & 20 & $17(85)$ & $3(15)$ & $1.9(0.4-7.7)$ & 0.52 & \\
\hline & No & 51 & $38(75)$ & $13(25)$ & & & \\
\hline \multirow[t]{2}{*}{ Previous antibiotic use } & Yes & 23 & $18(78)$ & $5(22)$ & $1.0(0.3-3.5)$ & 1.00 & \\
\hline & No & 48 & $37(77)$ & $11(23)$ & & & \\
\hline \multirow[t]{2}{*}{ S. aureus colonization } & Yes & 39 & $30(77)$ & $9(23)$ & $0.9(0.3-2.8)$ & 1.00 & \\
\hline & No & 32 & $25(78)$ & $7(22)$ & & & \\
\hline \multirow[t]{2}{*}{ Vaccine serotypes } & Yes & $28^{\mathrm{a}}$ & $25(89)^{\mathrm{a}}$ & $3(11)$ & $3.5(0.8-14.1)$ & 0.07 & 0.31 \\
\hline & No & $37^{\mathrm{a}}$ & $26(70)^{\mathrm{a}}$ & $11(30)$ & & & \\
\hline \multirow[t]{3}{*}{ SE } & High & 5 & $5(100)$ & $0(0)$ & NA & $0.02^{\mathrm{b} *}$ & 0.42 \\
\hline & Medium & 57 & $46(81)$ & $11(19)$ & & & \\
\hline & Low & 9 & $4(44)$ & $5(56)$ & & & \\
\hline
\end{tabular}

CI: confidence interval; OR: odds ratio; RI: respiratory infection; SE: socioeconomic status; UA: univariate analysis (Fisher's exact test); MA: multivariate analysis(logistic binary regression analysis)

${ }^{\text {a }}$ Numbers and percentages are based on the 65 S. pneumoniae strains available for serotyping

${ }^{\mathrm{b}}$ Pearson's Chi-square statisticunivariate analysis

*Significance: $\alpha=0.05$ 
(Table 4). In contrast, children with non-respiratory atopies and with a high socioeconomic status were invariably sparsely colonized. According to the univariate analyses, children with siblings $\leq 5$ years old and a low socioeconomic status were more densely colonized with $S$. pneumoniae $(p=0.047$ and $p=0.02$, respectively; Table 4 ). According to multivariate analyses, only having siblings $\leq 5$ years old remained a factor positively associated with dense pneumococcal colonization ( $p=0.02$; Table 4).

The prevalence of antimicrobial non-susceptibility among S. pneumoniae strains was as follows: trimethoprimsulfamethoxazole $40 \%$, erythromycin $38 \%$, penicillin $34 \%$ (intermediate $20 \%$ and high level 14\%), tetracycline $34 \%$, clindamycin $29 \%$, cefuroxime $15 \%$, meropenem $15 \%$, chloramphenicol $9 \%$, and ceftriaxone 5\% (Table 5). Resistance to cefotaxime, cefepime, and ertapenem was observed in $2 \%$ of the strains. We found no $S$. pneumoniae strains showing resistance to daptomycin, levofloxacin, moxifloxacin, linezolid, telithromycin, or vancomycin. Thirty-five percent of the $S$. pneumoniae strains $(n=23)$ were MDR (Table 5).

The serotypes observed among the 22 penicillin-nonsusceptible $S$. pneumoniae (PNSP) strains were 6B (40.9\%; $n=9), 23 \mathrm{~F}(22.7 \% ; n=5), 6 \mathrm{~A}(9.0 \% ; n=2), 19 \mathrm{~F}(9.0 \% ; n=2)$, and $14(4.5 \% ; n=1)$. The serotypes among the $23 \mathrm{MDR}$ strains were 6B $(39 \% ; n=9), 23 \mathrm{~F}(22 \% ; n=5), 14(13 \%$; $n=3), 23 \mathrm{~A}(13 \% ; n=3)$, and 19F $(4 \% ; n=1)$. Interestingly, $14 \%(n=3)$ of the PNSP and 9\% $(n=2)$ of the MDR strains were non-typeable. Overall, the prevalence of nonsusceptibility to any of the antimicrobials tested and of multidrug resistance was significantly increased among strains belonging to vaccine serotypes compared to strains belonging to non-vaccine serotypes ( $p$-values $<0.001$; Table 5).

Risk factors for colonization with trimethoprimsulfamethoxazole- or tetracycline-non-susceptible strains were allergies $[p=0.03$; OR (CI 95\%) $3.38(1.1-9.7)]$ and having siblings $[p=0.003$; OR (CI 95\%) $0.13(0.0-0.5)]$, respectively (data not shown). We found no clinical or demographic characteristics associated with non-susceptibility to any other antimicrobial drug studied. Interestingly, we observed a significant relationship between trimethoprimsulfamethoxazole non-susceptibility or multidrug resistance and S. pneumoniae colonization density. The vast majority of the trimethoprim-sulfamethoxazole-non-susceptible or MDR $S$. pneumoniae strains colonized children sparsely $(92 \%, p=$ 0.03 , and $96 \%, p=0.01$, respectively; Table 5).

\section{Discussion}

The prevalence of $S$. pneumoniae colonization reported in different parts of the world varies widely. This likely reflects both variation in study populations with respect to age, ethnicity, and socioeconomic conditions, as well as differences in sampling and isolation techniques. Moreover, the distribution of serotypes is temporal and varies by geographic location [2-4]. In the current study, we observed a pneumococcal colonization prevalence of $28 \%$ in Merida, Venezuela. In 2000, we studied a group of 125 healthy children under 5 years old from the same city, and we observed a similar colonization prevalence, i.e., $24 \%$ [19]. Previous studies performed in Latin America among healthy children in the same age group have shown significant differences of at least $37 \%$ in the prevalence of S. pneumoniae colonization, e.g., $21.4 \%$ in Mexico, $35.8 \%$ in Brazil, $41 \%$ and $48.3 \%$ in Peru, and $59.1 \%$ in Guatemala [25-28].

In the current study, serotypes $6 \mathrm{~B}, 19 \mathrm{~F}, 23 \mathrm{~F}, 15,6 \mathrm{~A}, 11$, $23 \mathrm{~A}$, and 34 were observed most frequently, accounting for $75 \%$ of all isolates. Except for serotype 34 , these serotypes have also been described as the most common serotypes colonizing children in other Latin American countries. Serotype 34 has been observed to colonize Peruvian children, albeit in a low proportion $[19,25-28]$. Of the $S$. pneumoniae strains isolated in our study, 51\% showed serotypes causing invasive diseases in Venezuela and 18 other Latin American countries [29]. We also observed one strain belonging to the recently described serotype $6 \mathrm{C}$, which has not been reported previously among carriers in Latin America. Although we did not isolate strains belonging to the heptavalent conjugate vaccine serotypes $4,9 \mathrm{~V}$, and $18 \mathrm{C}$, the other four serotypes included in this vaccine, i.e., $6 \mathrm{~B}, 14,19 \mathrm{~F}$, and $23 \mathrm{~F}$, were displayed by $43 \%$ of the strains. The distribution of serotypes among children in Venezuela has been previously described among the population of Warao Amerindians. This study revealed a comparable distribution of serotypes and frequency of vaccine serotypes [30]. Considering that the pneumococcal vaccine is not included in the vaccination schedule for healthy Venezuelan children, our study provides relevant information that will be useful for future monitoring of the effect of pneumococcal vaccination on the prevalence of colonization and the distribution of serotypes in this community.

Various clinical and demographic characteristics have been described to be associated with an increase in $S$. pneumoniae colonization, such as ethnicity, crowding, family size, siblings, smoking (passive and active), recent antibiotic use, and low income of the parents [4, 31], but none of those factors were found to be associated with nasal $S$. pneumoniae colonization in our study. However, our semiquantitative measurement of the density of colonization with $S$. pneumoniae showed that children with siblings $\leq 5$ years old were more densely colonized with $S$. pneumoniae. This is of great relevance, because exposure to other children during childhood, especially to younger siblings, has been clearly associated with an increased risk for invasive pneumococcal disease [31]. We also observed that colonization with 
Table 5 Frequency of antimicrobial resistance among $S$. pneumoniae strains according to vaccine serotypes and colonization density

\begin{tabular}{|c|c|c|c|c|c|c|c|c|c|c|c|c|c|c|c|}
\hline \multirow[t]{3}{*}{ Antimicrobial } & & \multirow{2}{*}{\multicolumn{2}{|c|}{ Strains, total }} & \multicolumn{4}{|c|}{ Serotypes } & \multirow[t]{3}{*}{ OR $(95 \% \mathrm{CI})^{\mathrm{a}}$} & \multirow[t]{3}{*}{$p$} & \multicolumn{4}{|c|}{ Colonization density } & \multirow[t]{3}{*}{ OR $(95 \% \mathrm{CI})^{\mathrm{a}}$} & \multirow[t]{3}{*}{$p$} \\
\hline & & & & \multicolumn{2}{|c|}{$\begin{array}{l}\text { Vaccine: } \\
n=28\end{array}$} & \multicolumn{2}{|c|}{$\begin{array}{l}\text { Non-vaccine: } \\
n=37\end{array}$} & & & \multicolumn{2}{|c|}{$\begin{array}{l}\text { Dense: } \\
n=14\end{array}$} & \multicolumn{2}{|c|}{$\begin{array}{l}\text { Sparse: } \\
n=51\end{array}$} & & \\
\hline & & $n$ & $\%$ & $n$ & $(\%)$ & $n$ & $(\%)$ & & & $n$ & $(\%)$ & $n$ & $(\%)$ & & \\
\hline \multirow[t]{2}{*}{$\mathrm{T} / \mathrm{S}$} & NS & 26 & 40 & 17 & $(65)$ & 9 & $(35)$ & $4.8(1.6-13.9)$ & $0.005 * *$ & 2 & $(8)$ & 24 & $(92)$ & $5.3(1.0-26.2)$ & $0.03 *$ \\
\hline & $\mathrm{S}$ & 39 & & 11 & & 28 & & & & 12 & & 27 & & & \\
\hline \multirow[t]{2}{*}{ Erythromycin } & NS & 25 & 38 & 20 & $(80)$ & 5 & $(20)$ & $16.0(4.5-55.8)$ & $0.000 * *$ & 2 & $(8)$ & 23 & $(92)$ & $4.9(1.0-24.2)$ & 0.06 \\
\hline & $\mathrm{S}$ & 40 & & 8 & & 32 & & & & 12 & & 28 & & & \\
\hline \multirow[t]{2}{*}{ PNSP } & NS & 22 & 34 & 17 & (77) & 5 & (23) & $9.8(2.9-33.1)$ & $0.000 * *$ & 4 & (18) & 18 & $(82)$ & $1.3(0.3-4.9)$ & 0.75 \\
\hline & $\mathrm{S}$ & 43 & & 11 & & 32 & & & & 10 & & 33 & & & \\
\hline \multirow[t]{2}{*}{ Tetracycline } & NS & 21 & 34 & 15 & (71) & 6 & (29) & $5.9(1.8-18.7)$ & $0.003 * *$ & 2 & $(10)$ & 19 & $(90)$ & $3.5(0.7-17.6)$ & 0.12 \\
\hline & $\mathrm{S}$ & 44 & & 13 & & 31 & & & & 12 & & 32 & & & \\
\hline \multirow[t]{2}{*}{ Clindamycin } & NS & 19 & 29 & 16 & (84) & 3 & (16) & $15.1(3.7-61.1)$ & $0.000 * *$ & 1 & (5) & 18 & (95) & $7.0(0.8-58.6)$ & 0.049 \\
\hline & $\mathrm{S}$ & 46 & & 12 & & 34 & & & & 13 & & 33 & & & \\
\hline \multirow[t]{2}{*}{ Cefuroxime } & NS & 11 & 15 & 11 & $(100)$ & 0 & $(0)$ & $3.1(2.1-4.7)$ & $0.000 * *$ & 1 & (9) & 10 & (91) & $3.1(0.3-27.1)$ & 0.43 \\
\hline & $\mathrm{S}$ & 54 & & 17 & & 37 & & & & 13 & & 41 & & & \\
\hline \multirow[t]{2}{*}{ Meropenem } & NS & 10 & 15 & 10 & $(100)$ & 0 & $(0)$ & $3.0(2.0-4.4)$ & $0.000 * *$ & 1 & (10) & 9 & $(90)$ & $2.7(0.3-24.0)$ & 0.67 \\
\hline & $\mathrm{S}$ & 55 & & 18 & & 37 & & & & 13 & & 42 & & & \\
\hline \multirow[t]{2}{*}{ Chloramphenicol } & NS & 6 & 9 & 6 & $(100)$ & 0 & $(0)$ & $2.0(1.9-3.7)$ & $0.005 * *$ & 0 & $(0)$ & 6 & (6) & $1.3(1.1-1.5)$ & 0.32 \\
\hline & $\mathrm{S}$ & 59 & & 22 & & 37 & & & & 14 & & 45 & & & \\
\hline \multicolumn{2}{|l|}{ MDR } & 23 & 35 & 18 & (78) & 5 & (2) & $11.5(3.4-38.9)$ & $0.0001 * *$ & 1 & (4) & 22 & (96) & $9.8(1.1-81.1)$ & $0.01 *$ \\
\hline \multicolumn{2}{|l|}{ Non-MDR } & 42 & & 10 & & 32 & & & & 13 & & 29 & & & \\
\hline
\end{tabular}

CI: confidence interval; OR: odds ratio; T/S: trimethoprim-sulfamethoxazole; PNSP: penicillin-non-susceptible S. pneumoniae; NS: nonsusceptible; S: susceptible; MDR: multidrug-resistant

${ }^{a}$ Univariate analysis(Fisher's exact test)

*Significance: $\alpha=0.05$

$* *$ Significance: $\alpha=0.01$

S. pneumoniae was progressively becoming denser as socioeconomic status decreased. We suspect that the combination of dense colonization and low socioeconomic status puts these children at increased risk to develop pneumococcal disease compared to the general population. In line with this, episodes of chronic otitis media with effusion have been related to an increased nasopharyngeal colonization density of S. pneumoniae [5], and several outbreaks of pneumococcal disease have occurred in communities suffering from poverty and malnutrition [32, 33]. Hence, pneumococcal conjugate vaccination is imperative in these groups of children.

Although day care center attendance has been associated with an increased risk for S. pneumoniae colonization [4], in our study, day care center attendees did not show an increased density of colonization nor an increased colonization rate. These findings might be explained by the low number of day care center attendees $(11 \%)$ present in our study, which affects the statistical power.

Within the past three decades, resistance of $S$. pneumoniae to $\beta$-lactams, macrolides, and other antimicrobial classes has escalated dramatically throughout the world. Great variation in the rates of resistant $S$. pneumoniae isolates from carriers have been observed between continents, countries, and even regions [6]. In most of the studies performed in Latin American children, resistance analysis was restricted to penicillin, and only in a few studies were trimethoprimsulfamethoxazole, erythromycin, chloramphenicol, and clindamycin investigated [6, 25-28, 34, 35]. In our study, resistance to trimethoprim-sulfamethoxazole was predominant (40\%), comparable to other Latin American countries where the resistance rates varied from $42 \%$ in Brazil [34] to $73 \%$ in Peru [27]. The prevalence of penicillin resistance has been reported to vary widely, for instance, ranging from $1.4 \%$ in the North region of Brazil to $49.0 \%$ in Fortaleza [6]. Likewise, high-level penicillin-resistance rates $(\geq 2 \mu \mathrm{g} / \mathrm{ml})$ ranged from $0 \%$ in certain regions of Brazil to $8 \%$ in Chile, but reached the exceptionally high rate of $38.8 \%$ observed among pneumococci colonizing children in Santa Fé, Argentina [6]. In our study, we observed alarmingly higher prevalence rates of penicillin- (34\%) and high-level 
penicillin-resistant strains (14\%) than those reported in most of the South American countries.

The described rates of non-susceptibility to other antimicrobials in Latin America also show a wide range: erythromycin from $7 \%$ in Peru [25] to $38 \%$ in Mexico [35]; clindamycin from $7 \%$ in Peru [27] to $18 \%$ in Brazil [34]; chloramphenicol from $0 \%$ in Mexico [28] to $27 \%$ in Peru [27]; and multidrug resistance from 3\% in Peru [27] to $20 \%$ in Guatemala [26]. Our results demonstrate one of the highest resistance rates against erythromycin (38\%), clindamycin (29\%), and multidrugs (35\%) among pneumococci colonizing healthy children in Latin American countries.

The highest global resistance is observed in those countries with easy access to antibiotics, such as Venezuela. Due to the continuous exposure of bacteria to antimicrobials, the selection of resistant strains occurs [7, 36]. Colonized children play an important role in the transmission of antibiotic-resistant pneumococcal strains within the community. Vaccination of children significantly reduces carriage and disease caused by antibiotic-resistant pneumococci in this population, as well as in unvaccinated children and adults due to herd immunity [36]. Our findings demonstrate a high prevalence of antimicrobial resistance among pneumococcal strains. Future efforts should be made to reduce the carriage and transmission of these strains, firstly by starting an appropriate pneumococcal vaccination program, and secondly by the substantial reduction of antimicrobial use and the use of antimicrobials that exert less selection pressure. These strategies will have the best impact on the control of pneumococcal antimicrobial resistance in the Venezuelan community [7, 36].

Trimethoprim-sulfamethoxazole-non-susceptible or MDR pneumococcal strains colonized children in a significantly less dense manner. Although antimicrobial resistance offers obvious advantages to bacteria, disadvantages such as a reduced virulence or viability might co-occur. In line with this, a lower prevalence of $S$. pneumoniae penicillin- or cephalosporin-resistant strains among isolates from blood or cerebrospinal fluid has been reported, explained by loss of fitness due to the acquisition of resistance [7]. We, therefore, hypothesize that certain trimethoprim-sulfamethoxazolenon-susceptible or MDR pneumococcal isolates may have a reduced ability to produce a dense nasal colonization due to a reduced ability to proliferate in the nasal mucosa.

To our knowledge, this study is the first assessment of nasal $S$. aureus colonization in healthy children in Venezuela. Recently, several studies have been conducted in Latin America to investigate the prevalence of $S$. aureus nasal colonization among healthy children. In children aged 2 months to 5 years attending day care centers in Brazil, and in Mexican children aged 6 months to 6 years, the prevalence of $S$. aureus colonization was $31.1 \%$ and $10.1 \%$, respectively $[37,38]$. Interestingly, while these rates were similar to those reported in other countries, such as Turkey (28.4\%) and the USA $(25.6 \%)[39,40]$, the prevalence of $S$. aureus among Venezuelan children was much higher $(56 \%)$.

With respect to risk factors, particular demographic characteristics have been related to nasal $S$. aureus colonization in children. For instance, in Brazilian children and in Turkish children, family size and educational level of the parents were associated with the prevalence of $S$. aureus nasal colonization [37, 39]. In addition, age, sex, and ethnicity were associated with nasal $S$. aureus colonization in the USA [40]. In our study, we found none of these demographic factors to be associated with $S$. aureus colonization.

Although an increase of $S$. aureus colonization among children with respiratory and non-respiratory allergies has been reported [11, 12], we observed no statistical differences in the prevalence of $S$. aureus colonization between children with asthma or rhinitis and children without these respiratory allergies. Instead, the only clinical characteristic positively associated with $S$. aureus colonization was nonrespiratory atopic disease. Similarly, several studies have also shown increased $S$. aureus nasal colonization among patients with atopic dermatitis compared to the general population [11, 41]. Super-antigens and toxins of $S$. aureus have been implicated as environmental factors in the pathogenesis of atopic dermatitis [41].

The only characteristic in our study that was negatively associated with $S$. aureus colonization was the nasal colonization by pneumococcal vaccine serotypes. Similar observations have been made for Dutch children aged 119 years, for Israeli children aged 40 months or younger, and for South African children aged 1-60 months [10, 16, 17]. It has been suggested that bacterial interference orchestrated by the host immune system might explain the inverse relation between $S$. aureus and $S$. pneumoniae [42, 43]. Our results demonstrate, for the first time, the negative association between these bacterial pathogens in the same niche, i.e., the nasal cavity. The introduction of pneumococcal conjugate vaccination is expected to reduce the morbidity and mortality caused by $S$. pneumoniae infections in our population. On the other hand, pneumococcal conjugate vaccination might alter the upper respiratory tract flora, and, consequently, increase the risk of $S$. aureus colonization and diseases [44]. To this respect, the development of a pneumococcal vaccine that only protects against invasive disease without affecting pneumococcal colonization is an interesting matter of scientific debate [44].

In summary, we observed that healthy Venezuelan children with young siblings and those of a low socioeconomic status were more densely colonized with $S$. pneumoniae. In addition, non-respiratory atopy was a risk factor for $S$. aureus colonization. Furthermore, $S$. pneumoniae vaccine serotypes 
were negatively associated with a previous history of RI and with $S$. aureus nasal colonization. Finally, a very high prevalence of pneumococcal multidrug-resistant strains among carriers was detected, and trimethoprim-sulfamethoxazoleand multidrug-resistant isolates were less densely present during colonization. These findings highlight the importance of the monitoring of colonization, and allow us to recommend the introduction of pneumococcal conjugate vaccination and to discuss the inappropriate antibiotic use in our community. This work provides a baseline for further assessment of epidemiological strategies aiming at the prevention and control of pneumococcal transmission, disease, and antimicrobial resistance.

Acknowledgments This study was partially financed by Los Andes University, Mérida, Venezuela, CDCHT No. M-813-04-07-C. We thank Dr. Moon Nahm and Dr. Jigui Yu, University of Alabama at Birmingham, AL, USA, for performing the characterization of the serotype $6 \mathrm{C}$ strain.

Open Access This article is distributed under the terms of the Creative Commons Attribution Noncommercial License which permits any noncommercial use, distribution, and reproduction in any medium, provided the original author(s) and source are credited.

\section{References}

1. Valenzuela MT, O'Loughlin R, De La Hoz F, Gomez E, Constenla D, Sinha A, Valencia JE, Flannery B, De Quadros CA (2009) The burden of pneumococcal disease among Latin American and Caribbean children: review of the evidence. Rev Panam Salud Pública 25(3):270-279

2. Greenberg D, Broides A, Blancovich I, Peled N, Givon-Lavi N, Dagan R (2004) Relative importance of nasopharyngeal versus oropharyngeal sampling for isolation of Streptococcus pneumoniae and Haemophilus influenzae from healthy and sick individuals varies with age. J Clin Microbiol 42(10):4604-4609

3. Rapola S, Salo E, Kiiski P, Leinonen M, Takala AK (1997) Comparison of four different sampling methods for detecting pharyngeal carriage of Streptococcus pneumoniae and Haemophilus influenzae in children. J Clin Microbiol 35(5):1077-1079

4. Bogaert D, De Groot R, Hermans PW (2004) Streptococcus pneumoniae colonisation: the key to pneumococcal disease. Lancet Infect Dis 4(3):144-154

5. García-Rodríguez JA, Fresnadillo Martínez MJ (2002) Dynamics of nasopharyngeal colonization by potential respiratory pathogens. J Antimicrob Chemother 50(Suppl S2):59-73

6. Cardozo DM, Nascimento-Carvalho CM, Souza FR, Silva NM (2006) Nasopharyngeal colonization and penicillin resistance among pneumococcal strains: a worldwide 2004 update. Braz J Infect Dis 10(4):293-304

7. Lynch JP 3rd, Zhanel GG (2009) Streptococcus pneumoniae: does antimicrobial resistance matter? Semin Respir Crit Care Med 30 (2):210-238

8. Wertheim HF, Melles DC, Vos MC, van Leeuwen W, van Belkum A, Verbrugh HA, Nouwen JL (2005) The role of nasal carriage in Staphylococcus aureus infections. Lancet Infect Dis 5(12):751-762

9. Peacock SJ, Justice A, Griffiths D, de Silva GD, Kantzanou MN, Crook D, Sleeman K, Day NP (2003) Determinants of acquisition and carriage of Staphylococcus aureus in infancy. J Clin Microbiol 41(12):5718-5725

10. Bogaert D, van Belkum A, Sluijter M, Luijendijk A, de Groot R, Rümke HC, Verbrugh HA, Hermans PW (2004) Colonisation by Streptococcus pneumoniae and Staphylococcus aureus in healthy children. Lancet 363(9424):1871-1872

11. Lomholt H, Andersen KE, Kilian M (2005) Staphylococcus aureus clonal dynamics and virulence factors in children with atopic dermatitis. J Invest Dermatol 125(5):977-982

12. Shiomori T, Yoshida S, Miyamoto H, Makishima K (2000) Relationship of nasal carriage of Staphylococcus aureus to pathogenesis of perennial allergic rhinitis. J Allergy Clin Immunol 105(3):449-454

13. Bassetti S, Sherertz RJ, Pfaller MA (2003) Airborne dispersal of Staphylococcus aureus associated with symptomatic rhinitis allergica. Ann Intern Med 139(3):W-W60

14. Bischoff WE, Wallis ML, Tucker BK, Reboussin BA, Pfaller MA, Hayden FG, Sherertz RJ (2006) "Gesundheit!" sneezing, common colds, allergies, and Staphylococcus aureus dispersion. J Infect Dis 194(8):1119-1126

15. Kenner J, O'Connor T, Piantanida N, Fishbain J, Eberly B, Viscount H, Uyehara C, Hospenthal D (2003) Rates of carriage of methicillinresistant and methicillin-susceptible Staphylococcus aureus in an outpatient population. Infect Control Hosp Epidemiol 24(6):439-444

16. McNally LM, Jeena PM, Gajee K, Sturm AW, Tomkins AM, Coovadia HM, Goldblatt D (2006) Lack of association between the nasopharyngeal carriage of Streptococcus pneumoniae and Staphylococcus aureus in HIV-1-infected South African children. J Infect Dis 194(3):385-390

17. Regev-Yochay G, Dagan R, Raz M, Carmeli Y, Shainberg B, Derazne E, Rahav G, Rubinstein E (2004) Association between carriage of Streptococcus pneumoniae and Staphylococcus aureus in children. JAMA 292(6):716-720

18. Pan American Health Organization (PAHO) (2007) Health situation in the Americas: basic indicators 2007. PAHO, Washington, DC. Available online at: http://www.paho.org/english/dd/ais/coredata.htm

19. Quintero B, Araque M (2006) Serotype profile and antibiotyping of Streptococcus pneumoniae strains isolated from nasal carriage in pediatric patients. Invest Clin 47(1):17-26

20. O’Brien KL, Bronsdon MA, Dagan R, Yagupsky P, Janco J, Elliott J, Whitney CG, Yang YH, Robinson LG, Schwartz B, Carlone GM (2001) Evaluation of a medium (STGG) for transport and optimal recovery of Streptococcus pneumoniae from nasopharyngeal secretions collected during field studies. J Clin Microbiol 39(3):1021-1024

21. Pai R, Gertz RE, Beall B (2006) Sequential multiplex PCR approach for determining capsular serotypes of Streptococcus pneumoniae isolates. J Clin Microbiol 44(1):124-131

22. Park IH, Park S, Hollingshead SK, Nahm MH (2007) Genetic basis for the new pneumococcal serotype, 6C. Infect Immun 75 (9):4482-4489

23. Park IH, Pritchard DG, Cartee R, Brandao A, Brandileone MC, Nahm MH (2007) Discovery of a new capsular serotype (6C) within serogroup 6 of Streptococcus pneumoniae. J Clin Microbiol 45(4):1225-1233

24. Clinical and Laboratory Standards Institute (CLSI) (2009) Performance Standards for Antimicrobial Susceptibility testing. Nineteenth Informational Supplement. CLSI Document M100-S19. CLSI, Wayne, PA

25. Cullotta AR, Kalter HD, Delgado J, Gilman RH, Facklam RR, Velapatino B, Coronel J, Cabrera L, Urbina M (2002) Antimicrobial susceptibilities and serotype distribution of Streptococcus pneumoniae isolates from a Low socioeconomic area in Lima, Peru. Clin Diagn Lab Immunol 9(6):1328-1331

26. Dueger EL, Asturias EJ, Matheu J, Gordillo R, Torres O, Halsey N (2008) Increasing penicillin and trimethoprim-sulfamethoxazole 
resistance in nasopharyngeal Streptococcus pneumoniae isolates from Guatemalan children, 2001-2006. Int J Infect Dis 12(3):289-297

27. Ochoa TJ, Rupa R, Guerra H, Hernandez H, Chaparro E, Tamariz J, Wanger A, Mason EO Jr (2005) Penicillin resistance and serotypes/ serogroups of Streptococcus pneumoniae in nasopharyngeal carrier children younger than 2 years in Lima, Peru. Diagn Microbiol Infect Dis 52(1):59-64

28. Solórzano-Santos F, Ortiz-Ocampo LA, Miranda-Novales MG, Echániz-Avilés G, Soto-Noguerón A, Guiscafré-Gallardo $\mathrm{H}$ (2005) Prevalence of Streptococcus pneumoniae serotypes on nasopharyngeal colonization in children of Mexico City. Salud Pública Méx 47(4):276-281

29. Gabastou JM, Agudelo CI, Brandileone MC, Castañeda E, de Lemos AP, Di Fabio JL (2008) Characterization of invasive isolates of $S$. pneumoniae, $H$. influenzae, and $N$. meningitidis in Latin America and the Caribbean: SIREVA II, 2000-2005. Rev Panam Salud Pública 24(1):1-15

30. Rivera-Olivero IA, Bogaert D, Bello T, del Nogal B, Sluijter M, Hermans PWM, de Waard JH (2007) Pneumococcal carriage among indigenous Warao children in Venezuela: serotypes, susceptibility patterns, and molecular epidemiology. Clin Infect Dis 45(11): 1427-1434

31. Hjuler T, Wohlfahrt J, Simonsen J, Kaltoft MS, Koch A, KamperJørgensen M, Biggar RJ, Melbye M (2007) Perinatal and crowding-related risk factors for invasive pneumococcal disease in infants and young children: a population-based case-control study. Clin Infect Dis 44(8):1051-1056

32. Dagan R, Gradstein S, Belmaker I, Porat N, Siton Y, Weber G, Janco J, Yagupsky P (2000) An outbreak of Streptococcus pneumoniae serotype 1 in a closed community in southern Israel. Clin Infect Dis 30(2):319-321

33. Romney MG, Hull MW, Gustafson R, Sandhu J, Champagne S, Wong T, Nematallah A, Forsting S, Daly P (2008) Large community outbreak of Streptococcus pneumoniae serotype 5 invasive infection in an impoverished, urban population. Clin Infect Dis 47(6):768-774

34. Rey LC, Wolf B, Moreira JL, Milatovic D, Verhoef J, Farhat CK (2002) Antimicrobial susceptibility and serotypes of nasopharyngeal Streptococcus pneumoniae in children with pneumonia and in children attending day-care centres in Fortaleza, Brazil. Int J Antimicrob Agents 20(2):86-92
35. Villaseñor-Sierra A, Lomas-Bautista M, Aguilar-Benavides S, Martínez-Aguilar G (2008) Serotypes and susceptibility of Streptococcus pneumoniae strains isolated from children in Mexico. Salud Pública Méx 50(4):330-333

36. Dagan R, Klugman KP (2008) Impact of conjugate pneumococcal vaccines on antibiotic resistance. Lancet Infect Dis 8 (12):785-795

37. Lamaro-Cardoso J, de Lencastre H, Kipnis A, Pimenta FC, Oliveira LS, Oliveira RM, Nouer SS, Aires-de-Sousa M, Milheiriço C, Andrade AL (2009) Molecular epidemiology and risk factors for nasal carriage of Staphylococcus aureus and methicillin-resistant $S$. aureus in infants attending day care centers in Brazil. J Clin Microbiol 47(12):3991-3997

38. Velázquez-Guadarrama N, Martínez-Aguilar G, Galindo JA, Zuñiga G, Arbo-Sosa A (2009) Methicillin-resistant S. aureus colonization in Mexican children attending day care centres. Clin Invest Med 32(1):E57-E63

39. Ciftci IH, Koken R, Bukulmez A, Ozdemir M, Safak B, Cetinkaya Z (2007) Nasal carriage of Staphylococcus aureus in 4-6 age groups in healthy children in Afyonkarahisar, Turkey. Acta Paediatr 96(7):1043-1046

40. Mainous AG 3rd, Hueston WJ, Everett CJ, Diaz VA (2006) Nasal carriage of Staphylococcus aureus and methicillin-resistant $S$ aureus in the United States, 2001-2002. Ann Fam Med 4 (2):132-137

41. Cork MJ, Robinson DA, Vasilopoulos Y, Ferguson A, Moustafa M, MacGowan A, Duff GW, Ward SJ, Tazi-Ahnini R (2006) New perspectives on epidermal barrier dysfunction in atopic dermatitis: gene-environment interactions. J Allergy Clin Immunol 118(1):321, quiz 22-23

42. Regev-Yochay G, Trzcinski K, Thompson CM, Malley R, Lipsitch M (2006) Interference between Streptococcus pneumoniae and Staphylococcus aureus: in vitro hydrogen peroxidemediated killing by Streptococcus pneumoniae. J Bacteriol 188 (13):4996-5001

43. Park B, Nizet V, Liu GY (2008) Role of Staphylococcus aureus catalase in niche competition against Streptococcus pneumoniae. J Bacteriol 190(7):2275-2278

44. Pettigrew MM, Gent JF, Revai K, Patel JA, Chonmaitree T (2008) Microbial interactions during upper respiratory tract infections. Emerg Infect Dis 14(10):1584-1591 